Supporting Information.

\title{
Room-Temperature Solution-Processed
}

\section{$\mathrm{NiO}_{\mathrm{x}}: \mathrm{PbI}_{2}$ Nanocomposite Structures for}

\section{Realizing High Performance Perovskite}

\section{Photodetectors}

Hugh Lu Zhu', Jiaqi Cheng ${ }^{\dagger}$, Di Zhang ${ }^{\S}{ }^{\dagger}$, Chunjun Liang', Claas J. Reckmeier ${ }^{\star}$, He Huang ${ }^{\not}$, Andrey L. Rogach ${ }^{+}$, Wallace C.H. Choy* ${ }^{*}$

${ }^{\dagger}$ Department of Electrical and Electronic Engineering, The University of Hong Kong, Pokfulam Road, Hong Kong, SAR China

${ }^{\ddagger}$ Department of Physics and Materials Science and Centre for Functional Photonics (CFP), City University of Hong Kong, Kowloon, Hong Kong, SAR China

$\S$ Department of Sustainable and Renewable Energy Engineering, University of Sharjah, United Arab Emirates

*E-mail: chchoy@eee.hku.hk (Wallace C.H. Choy). 

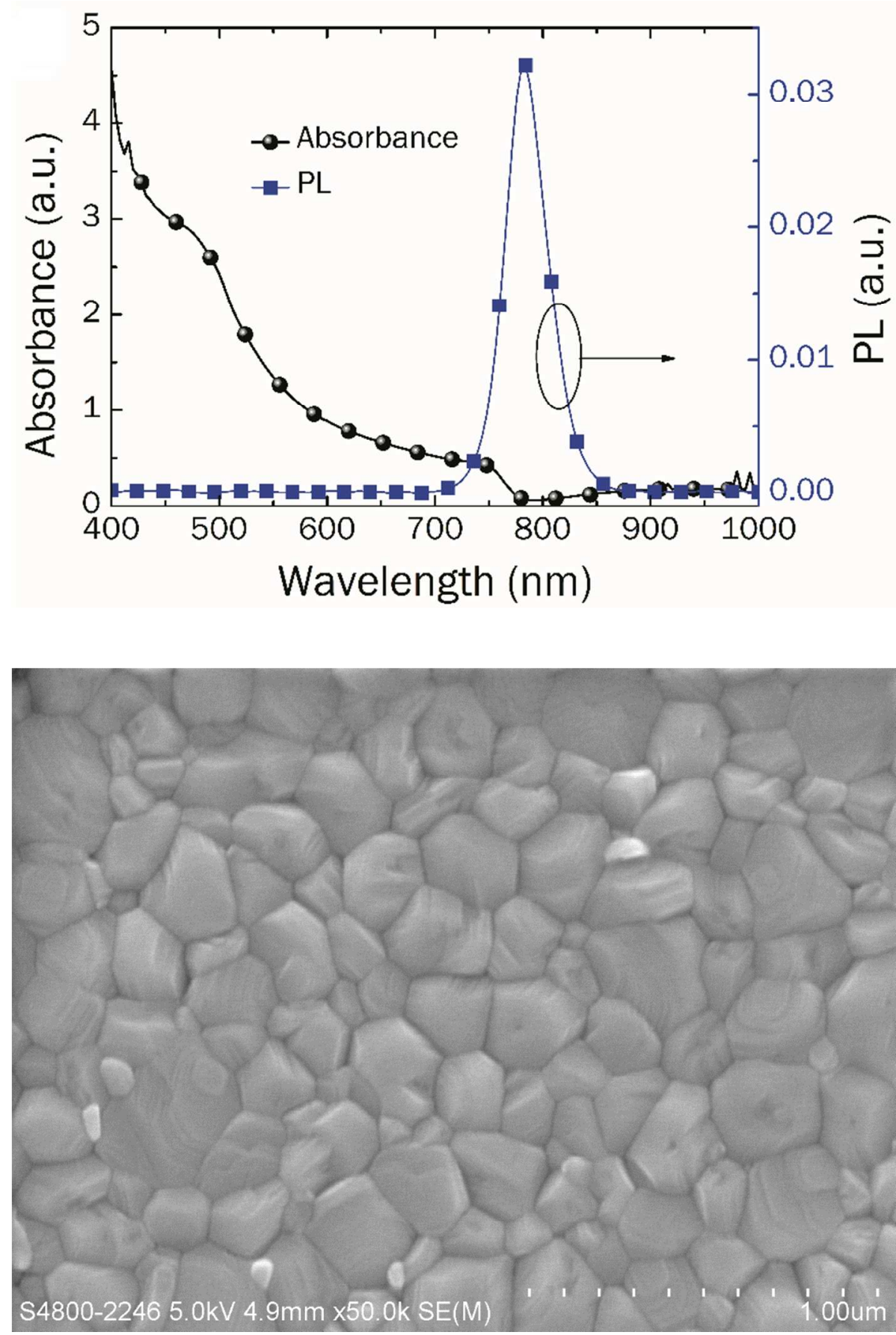

Figure S1. (Top) Absorbance and PL spectra of fabricated $\mathrm{MAPbI}_{3}$ polycrystalline films via the fast solution-processed sequential deposition. (Bottom) Top view SEM image of $\mathrm{MAPbI}_{3}$ polycrystalline films. 


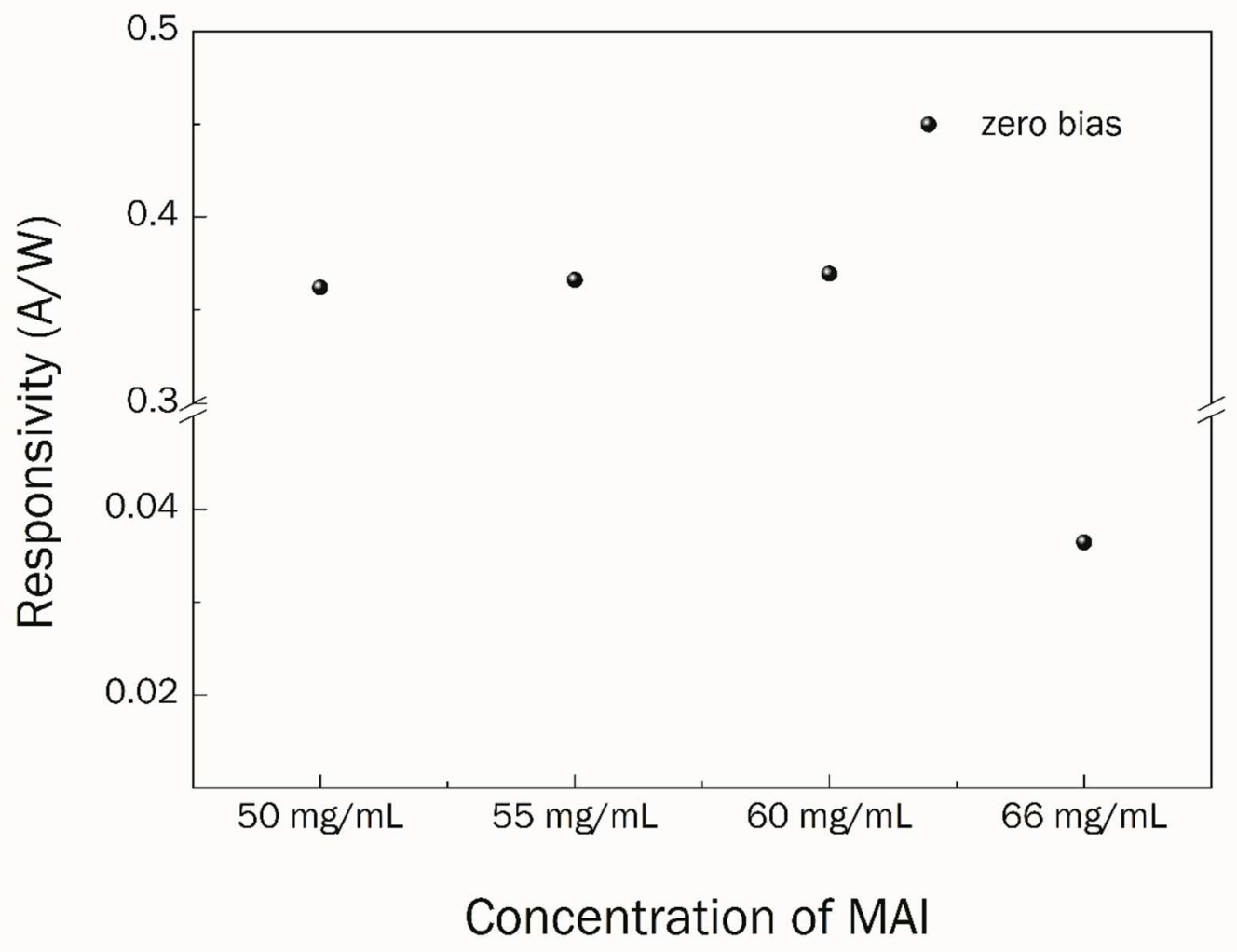

Figure S2. Responsivity of perovskite PDs illuminated by the incident light of $532 \mathrm{~nm}$ with different concentrations of MAI. 


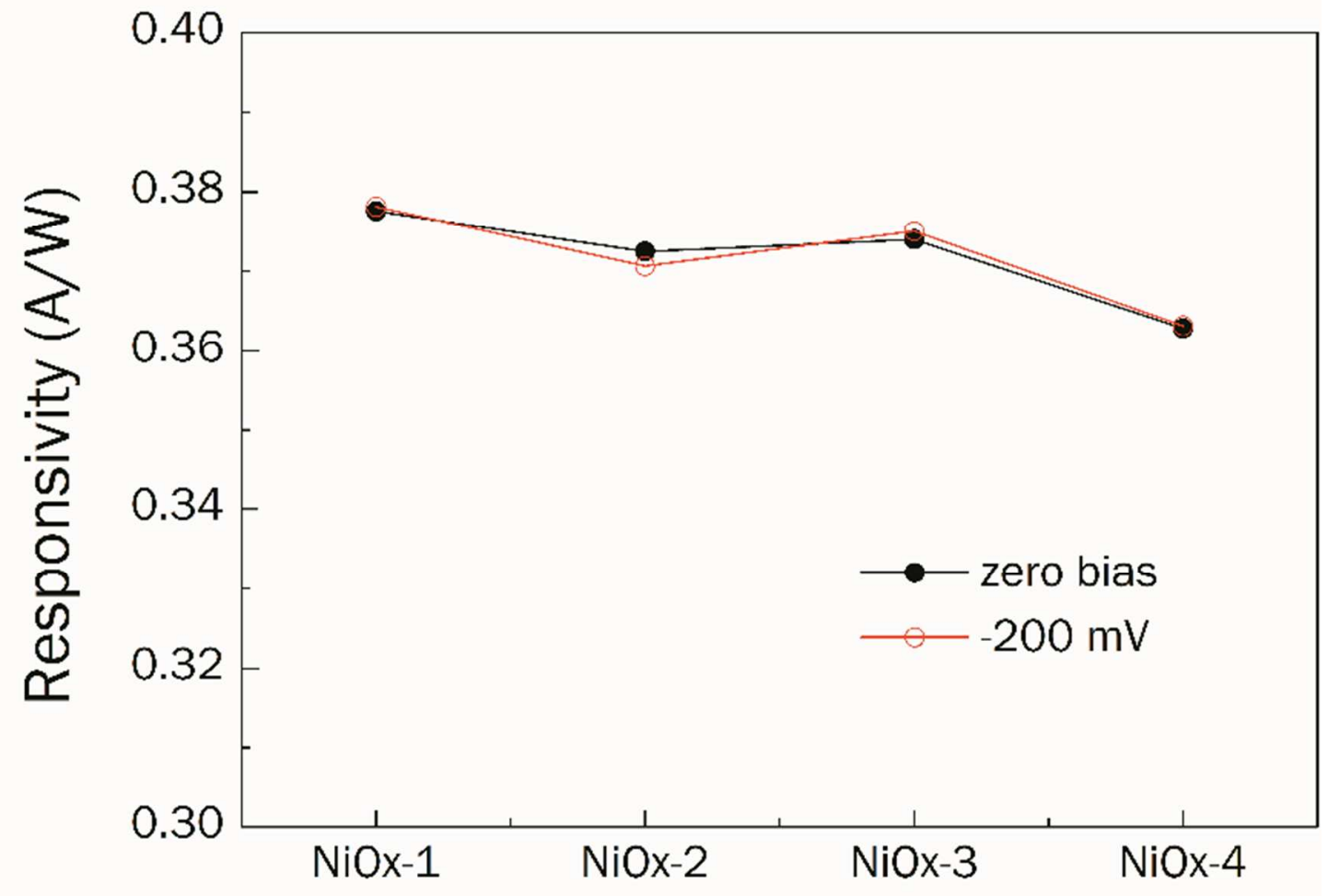

Figure S3. Responsivity of perovskite PDs illuminated by the incident light of $532 \mathrm{~nm}$ with four different thicknesses of $\mathrm{NiO}_{\mathrm{x}}$ HELs at zero bias and $-200 \mathrm{mV}$. The thickness of $\mathrm{NiO}_{\mathrm{x}}-1, \mathrm{NiO}_{\mathrm{x}}-2, \mathrm{NiO}_{\mathrm{x}}-3$ and $\mathrm{NiO}_{\mathrm{x}}-4$ is $18,15,14$ and $13 \mathrm{~nm}$, respectively. 


\section{$\mathrm{NiO}_{x}-1$}

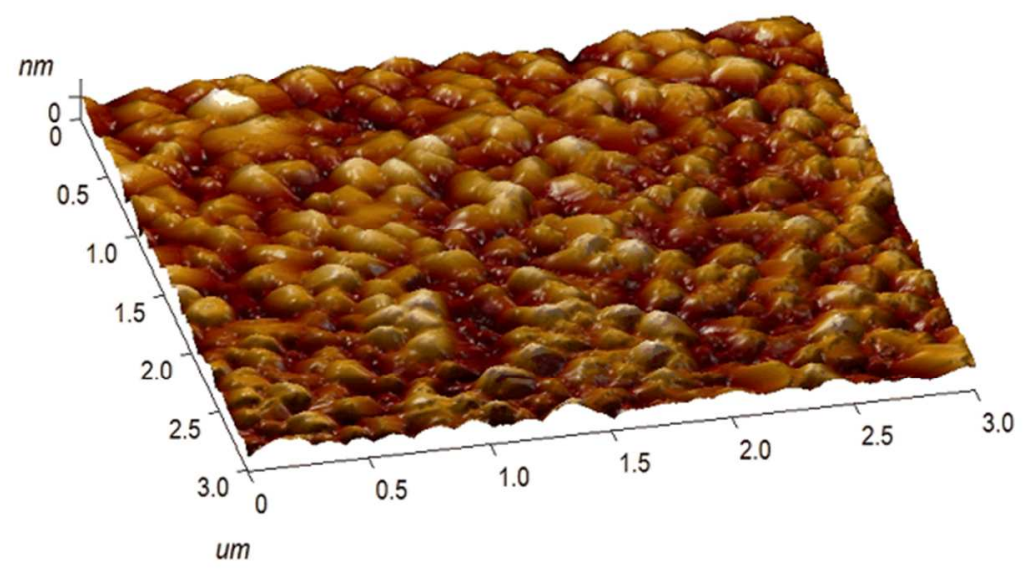

\section{$\mathrm{NiO}_{x}-4$}

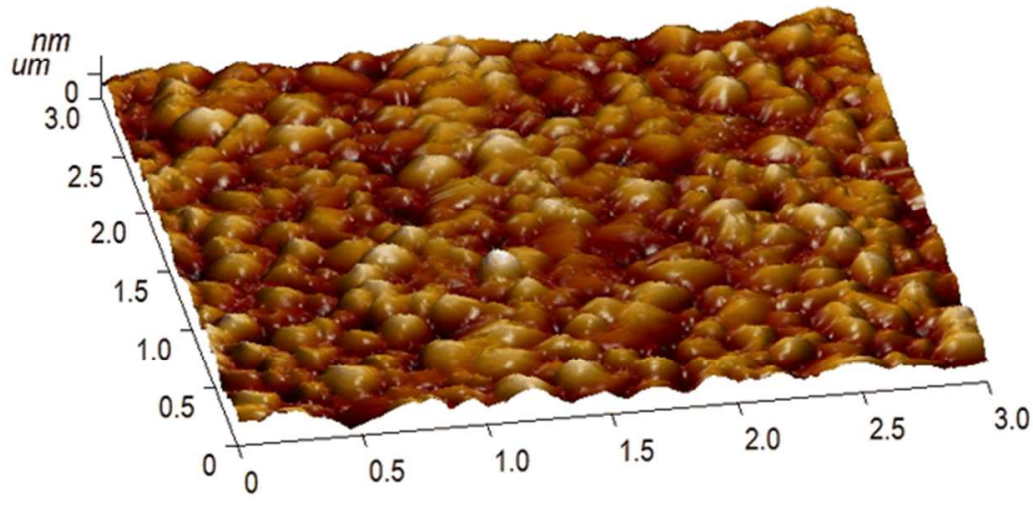

Figure S4. 3D view AFM images of $\mathrm{MAPbI}_{3}$ formed on $\mathrm{NiO}_{\mathrm{x}}-1$ and $\mathrm{NiO}_{\mathrm{x}}-4$. The roughness of $\mathrm{MAPbI}_{3}$ on $\mathrm{NiO}_{\mathrm{x}}-1$ and $\mathrm{NiO}_{\mathrm{x}}-4$ is 9.8 and $9.8 \mathrm{~nm}$, respectively. 


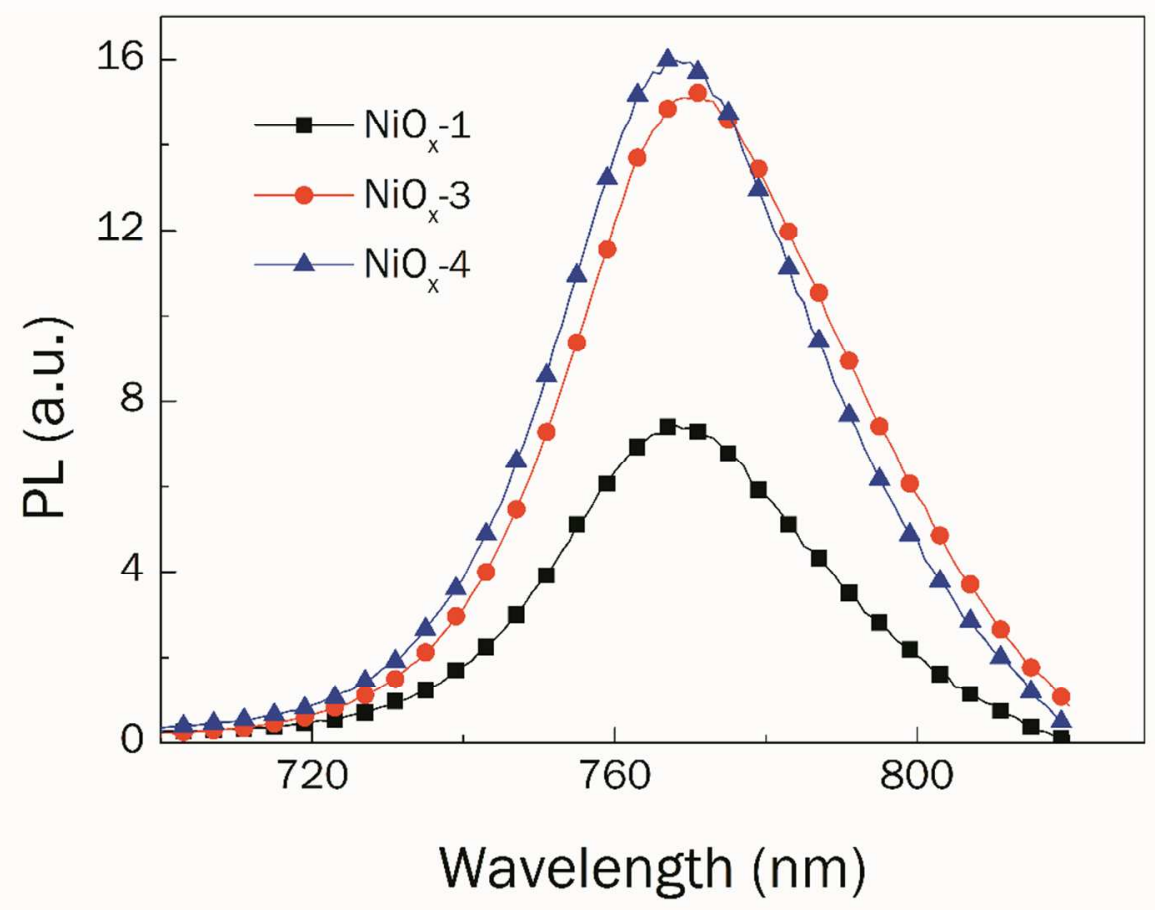

Figure S5. PL spectra of $\mathrm{MAPbI}_{3}$ perovskites grown on different thickness dependent $\mathrm{NiO}_{\mathrm{x}}$ substrates. 


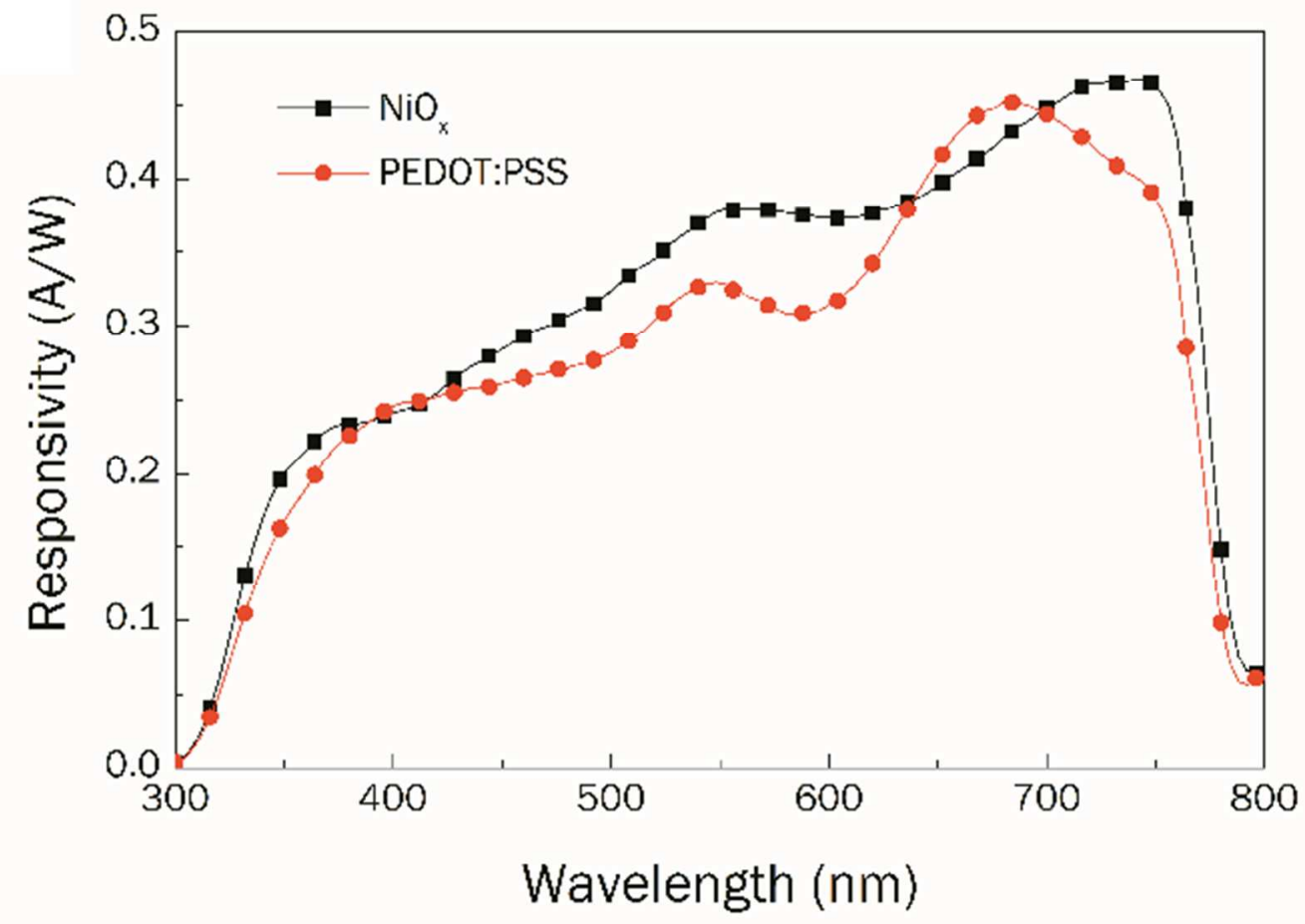

Figure S6. Spectral responsivity of PEDOT:PSS and $\mathrm{NiO}_{\mathrm{x}}$ based perovskite PDs, respectively. 


\section{Shot noise, thermal noise and saturation photocurrent}

Thermal noise current is expressed by $i_{\text {thermal }}=\left(4 k_{B} T \Delta f / R_{s h}\right)^{1 / 2}$ where $k_{B}$ is the Boltzmann constant, $T$ is the working temperature, $\Delta f$ is the bandwidth, $R_{s h}$ is the shunt resistance of PDs. Hence, leakage current induced shunt resistance $R_{s h}$ largely contributes to the thermal noise. Shot noise current is defined by $i_{\text {shot }}=\left(2 J_{\text {dark }} q \Delta f\right)^{1 / 2}$ where $J_{\text {dark }}$ is the dark current, $q$ is the elementary charge. Thus, $J_{\text {dark }}$ (reverse leakage current) largely originating from reverse bias induced charges injection from the external circuit makes a large contribution to the shot noise.

The saturation current is determined by space charge limited current, ${ }^{1,2}$ which is defined by $J_{S C L C}=9 \varepsilon \mu V^{2} / 8 L^{3}$, where $\varepsilon$ is the dielectric permittivity, $\mu$ is the slowest charge carrier mobility, $V$ is the voltage and $L$ is the thickness. 


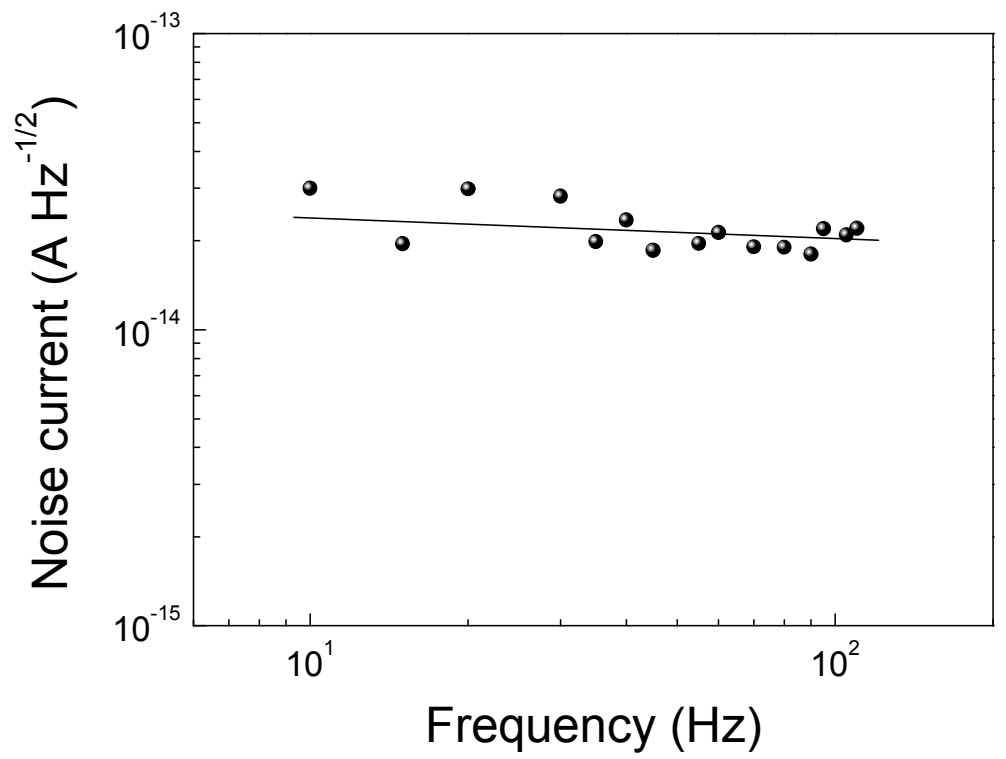

Figure S7. The measured noise current of perovskite PDs determined by Stanford Research SR830 Lock-in Amplifier in current measurement mode. The system input noise is $13 \mathrm{fA} \mathrm{Hz}^{-1 / 2}$. 
$\mathrm{C}_{60}$ electron mobility measurement

The electron mobility of $\mathrm{C}_{60}$ is measured by the space charge limited current (SCLC) technique with the device structure of $\mathrm{ITO} / \mathrm{TiO}_{2} / \mathrm{C}_{60} / \mathrm{Al}$. The fitting electron mobility of $\mathrm{C}_{60}$ is $1.11 \times 10^{-3} \mathrm{~cm}^{2} / \mathrm{Vs}$.

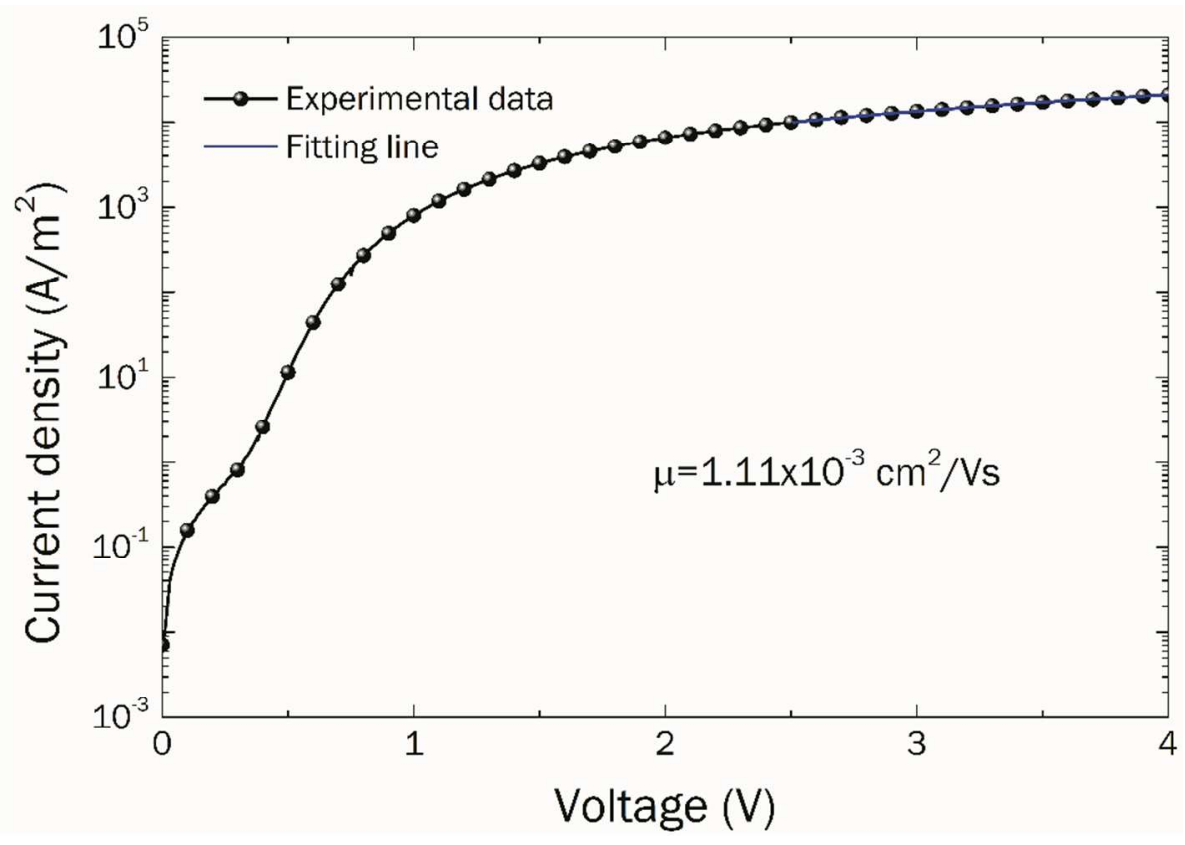

Figure S8. The electron mobility of $\mathrm{C}_{60}$ measured by the SCLC technique. 
Calculating carrier transit time of perovskite PDs

Using the perovskite thickness with $250 \mathrm{~nm}$, the carrier mobility with $8.1 \mathrm{~cm}^{2} / \mathrm{Vs},{ }^{3}$ the negative bias with $1 \mathrm{~V}$, thus the time of carrier transit across the perovskite layer is $77 \mathrm{ps}$. Using the $\mathrm{C}_{60}$ thickness with $50 \mathrm{~nm}$ (from cross-section SEM image in Figure 1(b)), the carrier mobility with $1.11 \times 10^{-3} \mathrm{~cm}^{2} / \mathrm{Vs}$ (extracting from the SCLC curve shown in Figure S8), ${ }^{4}$ the negative bias with $1 \mathrm{~V}$, thus the time of carrier transit across the $\mathrm{C}_{60}$ layer is 23 ns.

Therefore, the lowest carrier mobility of $\mathrm{C}_{60}$ determine carrier transit time within perovskite PDs. 


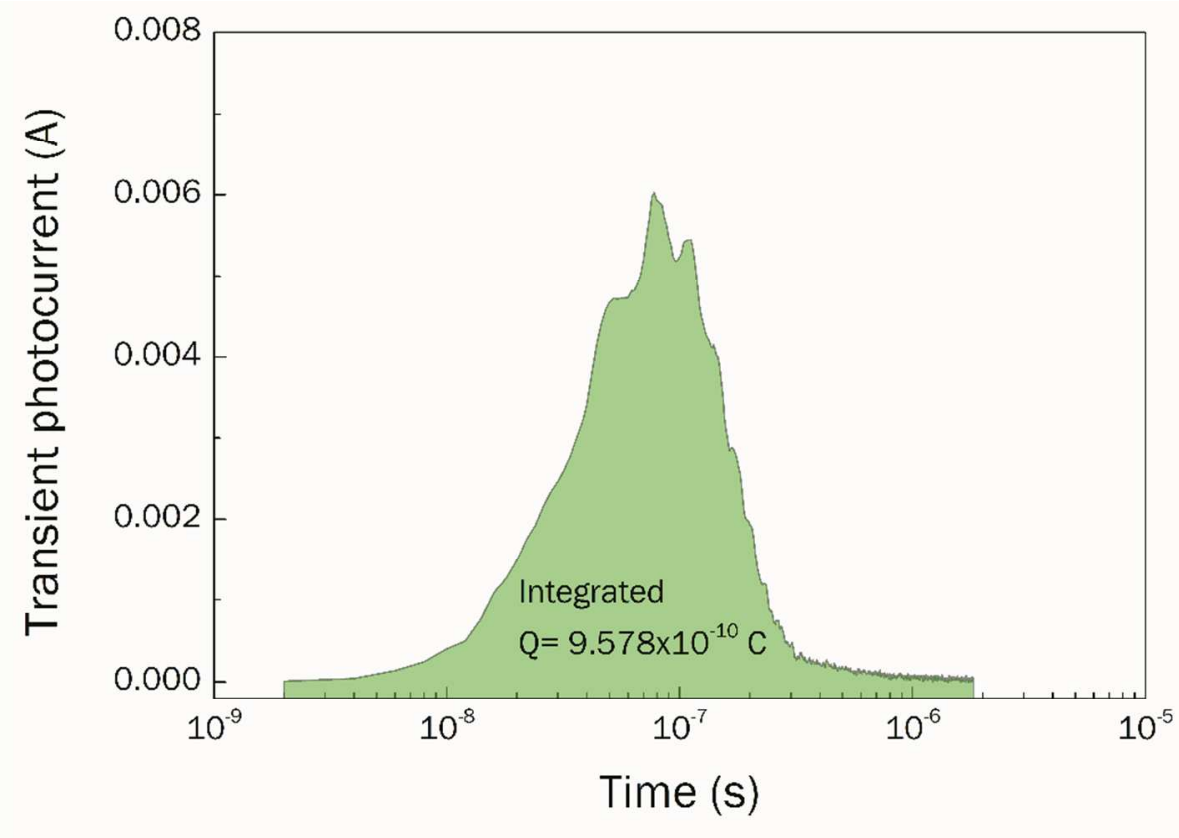

Figure S9. The photocurrent response of perovskite PDs under the illumination of $532 \mathrm{~nm}$ incident light. The capacitance $C$ of perovskite PDs is calculated by the following equation $C=Q / V$. Using intergrated $\mathrm{Q}=0.9578 \mathrm{nC}, \mathrm{V}=0.0595 \mathrm{~V}$, the calculated $\mathrm{C}=16.10$ $\mathrm{nF}$. Therefore, the RC time constant of PDs is $161 \mathrm{~ns}$. 


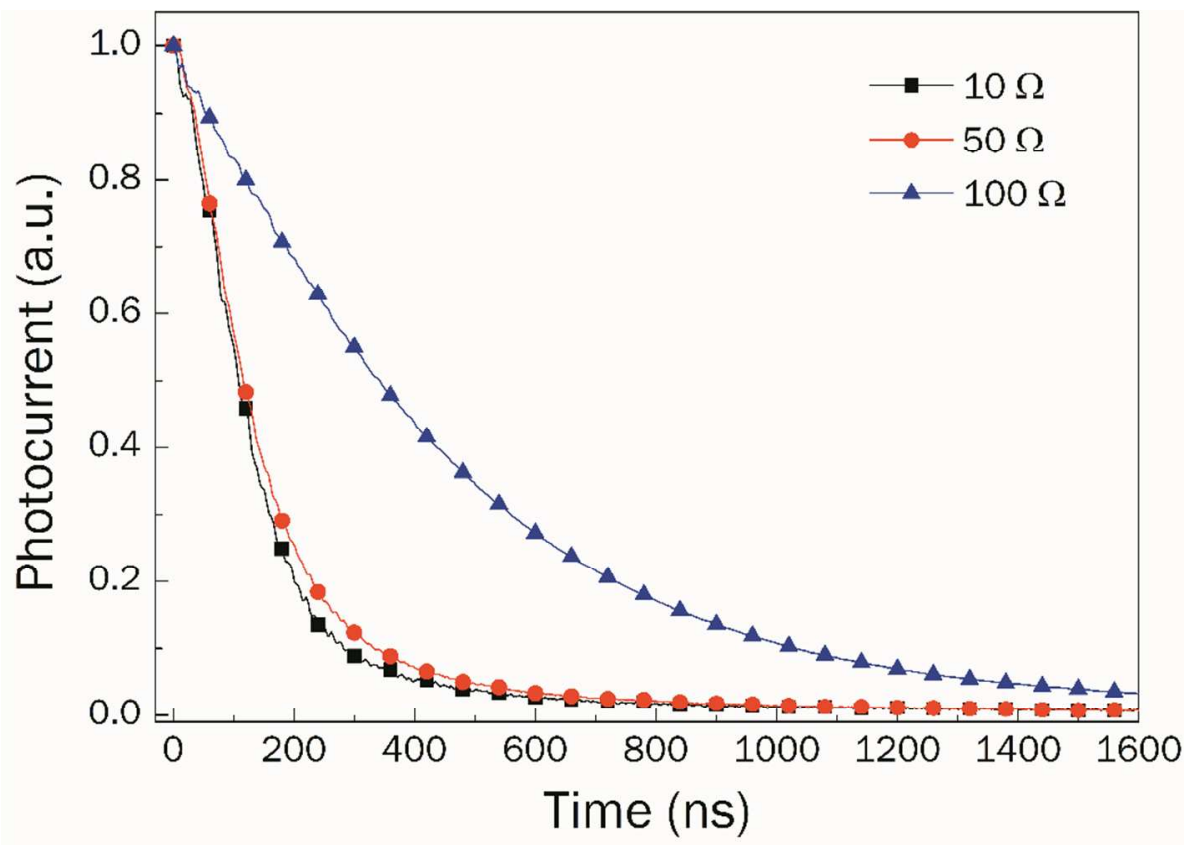

Figure S10. The transient photocurrent response of perovskite PDs as a function of three load resistors under the illumination of $532 \mathrm{~nm}$ incident light. It can be clearly seen that $\mathrm{RC}$ time constant limits the photocurrent response time of our proposed perovskite PDs, which confirms our above discussion. 

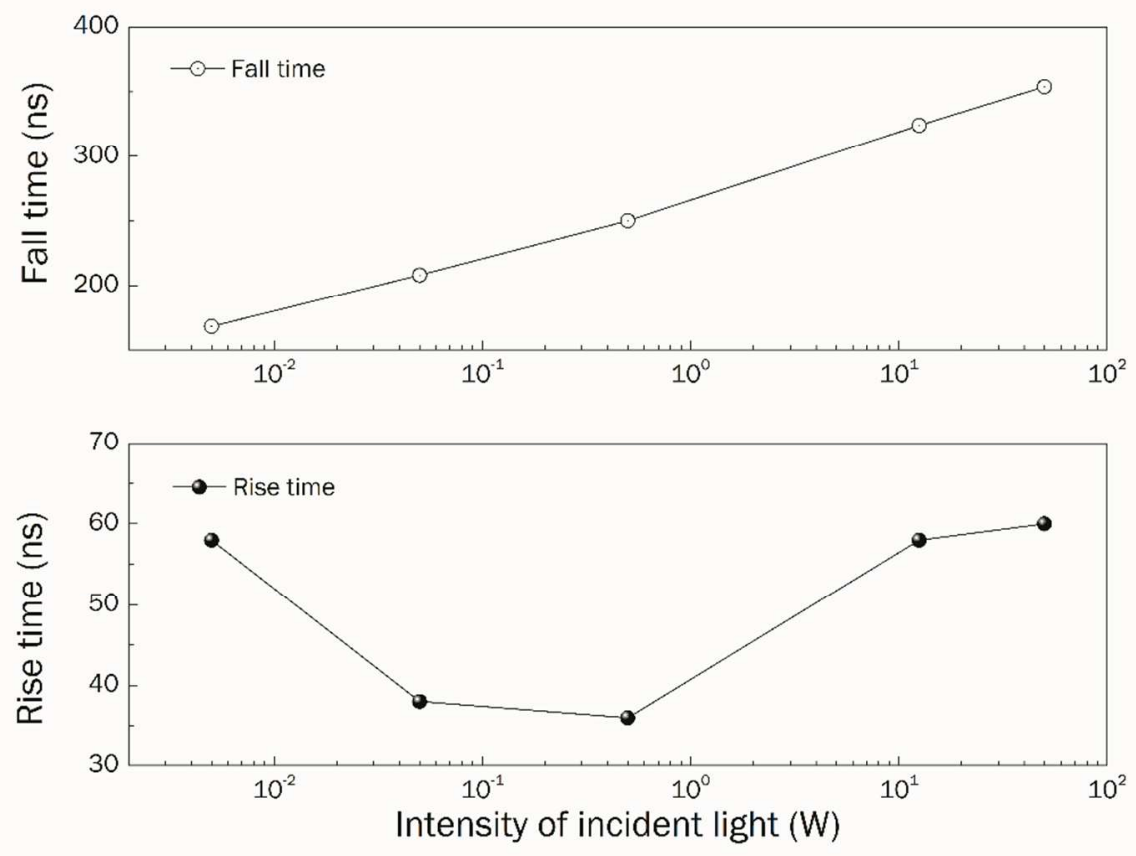

Figure S11. The rise time and fall time of perovskite PDs as a function of the intensity of $532 \mathrm{~nm}$ incident light. Due to RC limited photocurrent response time in perovskite PDs, the rise time and fall time of perovskite PDs basically obey the trend that they increase slowly with the increased intensity of incident light, suggesting that higher power intensity leads to raised capacitance. This is probably due to the enhanced dielectric constant of perovskite materials under the illumination of higher power intensity. 


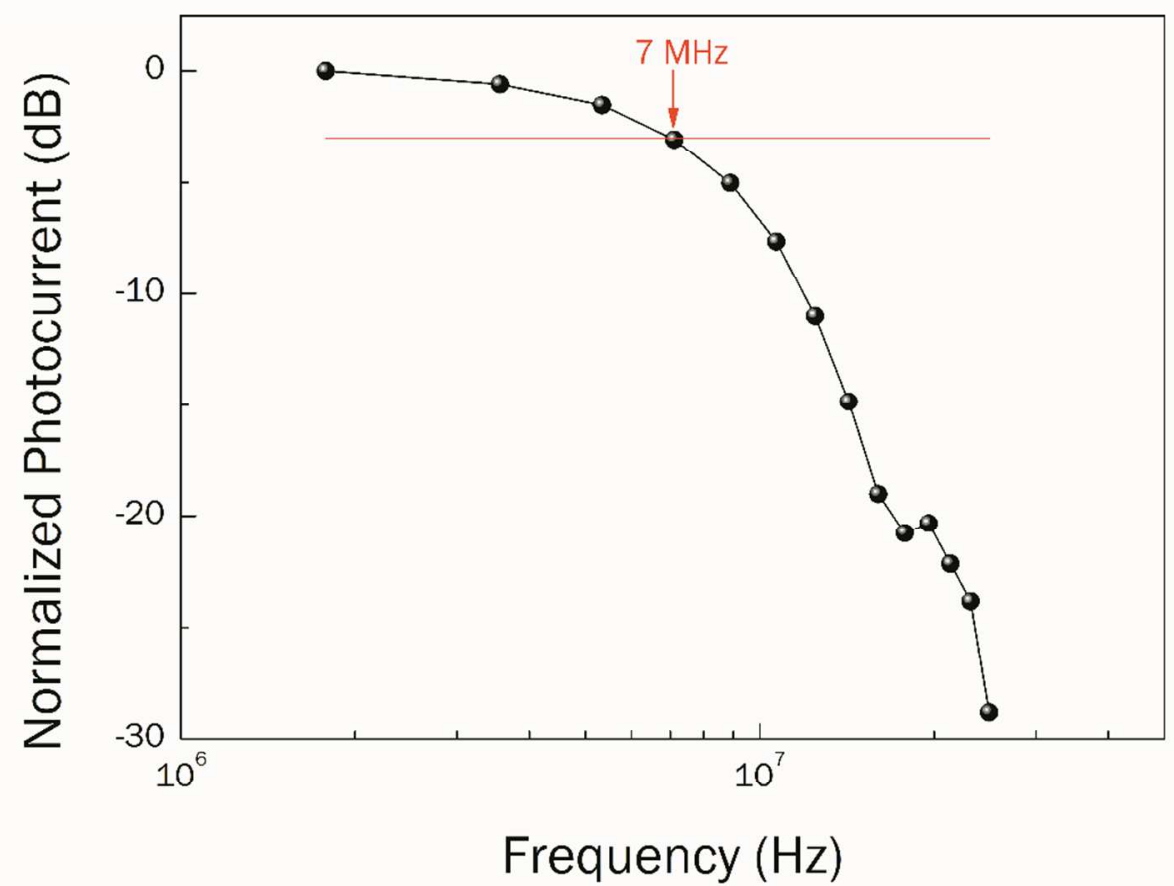

Figure S12. Normalized photocurrent response of perovskite PDs (with $\sim 1.6 \mathrm{~mm}^{2}$ ) versus the modulation frequency of incident light. The line of $-3 \mathrm{~dB}$ is depicted for reference. 


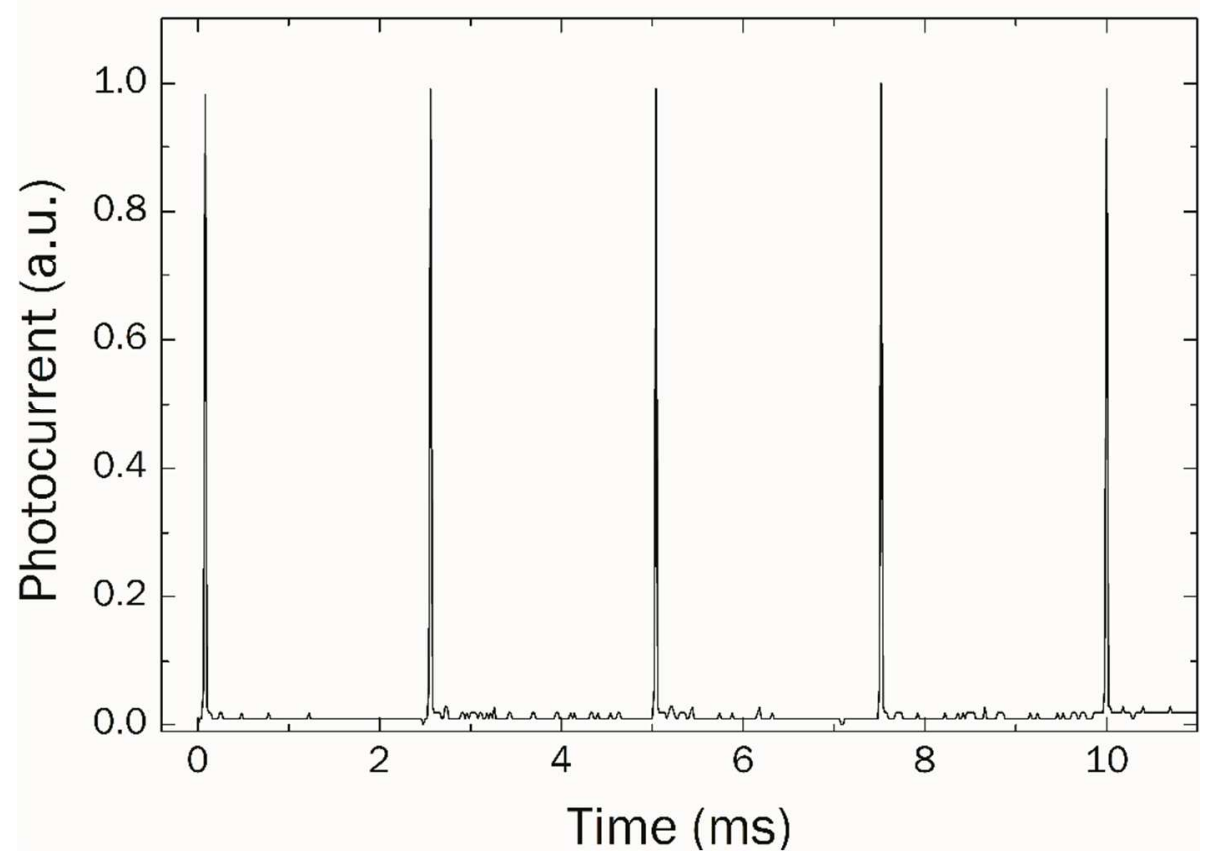

Figure S13. Repeatable photocurrent response of perovskite PDs at a pulse frequency of $400 \mathrm{~Hz}$. 


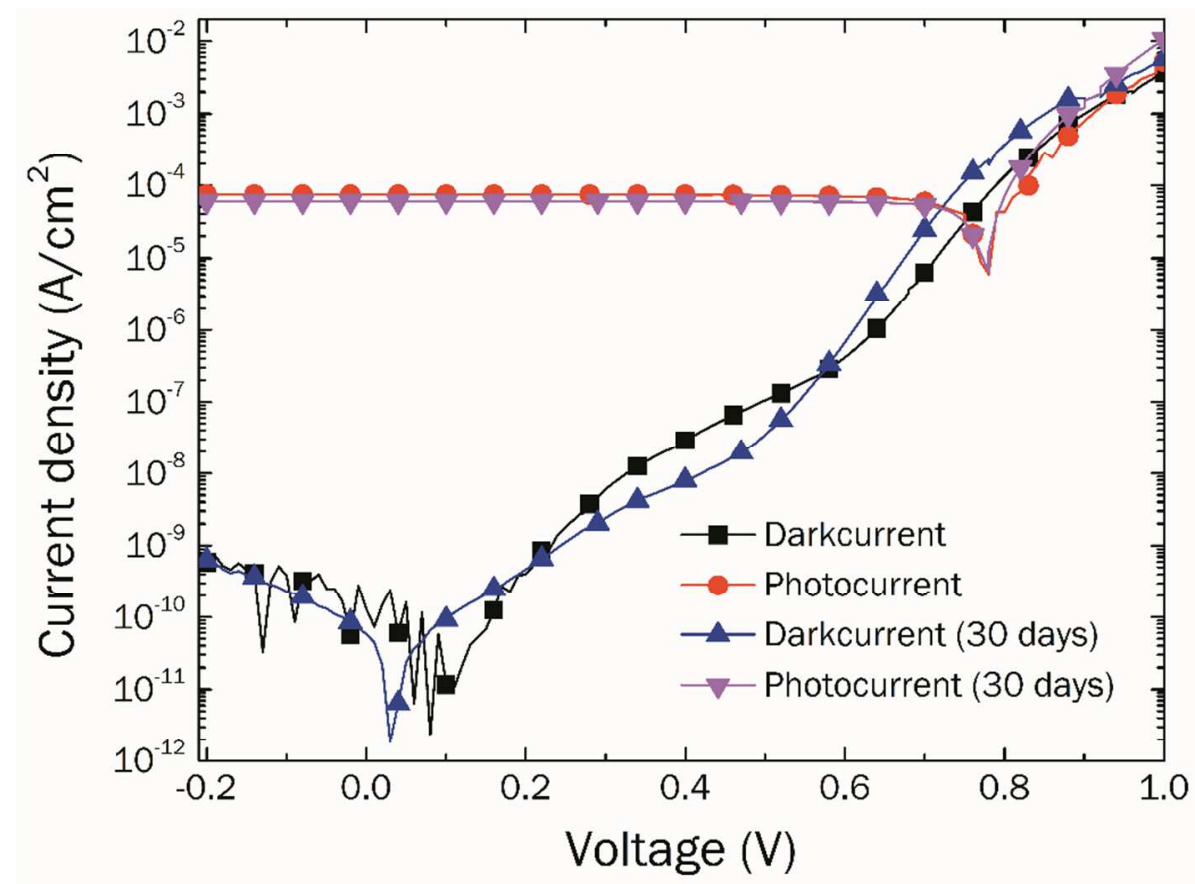

Figure S14. The stability of perovskite PDs. Photocurrent and dark current of perovskite PDs stored in vacuum were measured after 30 days.

\section{REFERENCES}

1. Goodman, A. M.; Rose, A., Double Extraction of Uniformly Generated Electron-Hole

Pairs from Insulators with Noninjecting Contacts. J. Appl. Phys. 1971, 42, 2823-2830.

2. Mihailetchi, V. D.; Wildeman, J.; Blom, P. W. M., Space-Charge Limited

Photocurrent. Phys. Rev. Lett. 2005, 94, 126602.

3. Wehrenfennig, C.; Eperon, G. E.; Johnston, M. B.; Snaith, H. J.; Herz, L. M., High

Charge Carrier Mobilities and Lifetimes in Organolead Trihalide Perovskites. Adv.

Mater. 2014, 26, 1584-1589. 
4. Sha, W. E. I.; Zhu, H. L.; Chen, L.; Chew, W. C.; Choy, W. C. H., A General Design Rule to Manipulate Photocarrier Transport Path in Solar Cells and Its Realization by the Plasmonic-Electrical Effect. Sci. Rep. 2015, 5, 8525. 